\title{
A Characteristic Division Between the Fueling of Quasars and Seyferts: Five Simple Tests
}

\section{Citation}

Hopkins, Philip F., and Lars Hernquist. 2009. "A CHARACTERISTIC DIVISION BETWEEN THE FUELING OF QUASARS AND SEYFERTS: FIVE SIMPLE TESTS." The Astrophysical Journal 694 (1): 599-609. https://doi.org/10.1088/0004-637x/694/1/599.

\section{Permanent link}

http://nrs.harvard.edu/urn-3:HUL.InstRepos:41381859

\section{Terms of Use}

This article was downloaded from Harvard University's DASH repository, and is made available under the terms and conditions applicable to Open Access Policy Articles, as set forth at http:// nrs.harvard.edu/urn-3:HUL.InstRepos:dash.current.terms-of-use\#OAP

\section{Share Your Story}

The Harvard community has made this article openly available.

Please share how this access benefits you. Submit a story.

Accessibility 
ACCePted to ApJ, DeCEMBer 14, 2008

Preprint typeset using LTEX style emulateapj v. 08/22/09

\title{
A CHARACTERISTIC DIVISION BETWEEN THE FUELING OF QUASARS AND SEYFERTS: FIVE SIMPLE TESTS
}

\author{
PHILIP F. HOPKINS ${ }^{1}$, LARS HERNQUIST ${ }^{1}$ \\ Accepted to ApJ, December 14, 2008
}

\begin{abstract}
Given the existence of the $M_{\mathrm{BH}}-\sigma$ relation, models of self-regulated black hole (BH) growth require both a fuel supply and concomitant growth of the host bulge to deepen the central potential, or else the system will either starve or immediately self-regulate without any sustained activity. This leads to a generic prediction that the brightest quasars must be triggered in major mergers: a large fraction of the galaxy mass must be added/converted to new bulge mass and a galactic supply of gas must lose angular momentum in less than a dynamical time. Low-luminosity active galactic nuclei (AGN), in contrast, require little bulge growth and small gas supplies, and could be triggered in more common non-merger events. This leads to the expectation of a characteristic transition to merger-induced fueling around the traditional quasar-Seyfert luminosity divide (growth of BH masses above/below $\sim 10^{7} M_{\odot}$ ). We compile and survey a number of observations in order to test several predictions of such a division, including: (1) A transition to bulge-dominated hosts (which any major merger remnant, regardless of difficult-to-observe tidal features, should be). (2) A transition between "pseudobulges" and "classical" bulges hosting the remnant BHs: pseudobulges are formed in secular processes and minor mergers, whereas classical bulges are relics of major mergers. (3) An increase in the amplitude of small-scale clustering (increased halo occupation of small group environments) where mergers are more efficient. (4) Different redshift evolution, with gas-rich merger rates rising to redshifts $z>2$ while secular processes are relatively constant in time. (5) An increasing prominence of post-starburst features in more luminous systems. Our compilation of observations in each of these areas provides tentative evidence for the predicted division around the Seyfert-quasar threshold, and we discuss how future observations can improve these constraints and, in combination with the tests here, break degeneracies between different fueling models. Subject headings: quasars: general — galaxies: active — galaxies: evolution — cosmology: theory
\end{abstract}

\section{INTRODUCTION}

The discovery of tight correlations between black hole (BH) mass and properties of the host galaxy spheroid, including spheroid luminosity (Kormendy \& Richstone 1995), stellar mass (Magorrian et al. 1998), velocity dispersion (Ferrarese \& Merritt 2000; Gebhardt et al. 2000), concentration and profile shape (Graham et al. 2001; Graham \& Driver 2007), have fundamental implications for the growth of black holes and - given the Soltan (1982) argument which implies that most black hole mass was assembled in luminous quasar phases (for more recent versions of this calculation, see e.g. Salucci et al.| 1999; Yu \& Tremaine 2002; Hopkins et al. 2007d; Shankar et al. 2007) - corresponding quasar activity.

In order to explain not just the normalization but also the small (factor $\lesssim 2$ ) observed scatter in these correlations (Tremaine et al. 2002), models generically require some sort of self-regulated growth (e.g. Silk \& Rees 1998; Burkert \& Silk 2001; Wyithe \& Loeb 2002; Di Matteo et al. 2005; Hopkins et al. 2005a, 2007b a; Murray et al. 2005; Thompson et al. 2005). Because the BH represents such a small fraction $\left(\sim 10^{-3}\right)$ of the galaxy mass, it is difficult (if not impossible) to avoid at least the occasional rare situation in which $\gtrsim 100$ times the implied $\mathrm{BH}$ mass, from e.g. the $M_{\mathrm{BH}}-\sigma$ relation, in cold gas is stripped of angular momentum and should accrete rapidly (given either e.g. Bondi or thin-disk accretion), giving rise to BHs well off the observed correlation and orders of magnitude outside the scatter. This concern has led models to invoke some form of feedback from the $\mathrm{BH}$ as a self-regulation mechanism: regardless of the de-

\footnotetext{
${ }^{1}$ Harvard-Smithsonian Center for Astrophysics, 60 Garden Street, Cambridge, MA 02138
}

tails, once the $\mathrm{BH}$ reaches some critical mass, if a small fraction $(\lesssim 5 \%)$ of the radiant energy $(\sim 0.5 \%$ of the accretion energy) or momentum can couple to the nearby gas, then simple scalings show that gas will be unbound and not able to accrete. The equilibrium between the coupled energy/momentum and the depth of the central potential therefore sets the maximum black hole mass.

In addition to the nature and small scatter of the observed BH-host correlations, there is increasing evidence for a process of this nature. Hopkins et al. (2007b a) pointed out that these scenarios generically imply that the most basic correlation is really between $\mathrm{BH}$ mass and central potential depth/spheroid binding energy, and demonstrated in the observations $\mathrm{a} \sim 3 \sigma$ preference for such an association (i.e. the only relationship with no residual correlations remaining between $\mathrm{BH}$ mass and other host galaxy properties). Aller \& Richstone (2007) found a similar result in an independent analysis of the observations modeling the central potential in generality, including the contribution of disks and halos.

Moreover, although it remains unclear which feedback processes are the most important for this self-regulation, numerous such mechanisms are observed to be ubiquitous in quasar populations, including intermediate-scale winds, accretion disk outflows, jets, ionization fronts, Compton heating, radiation pressure, and shocks (see e.g. Weymann et al. 1981; Stark \& Carlson 1984; Baum et al. 1993; Colbert et al. 1996b. a, 1998; Laor et al. 1997; Crenshaw et al. 2000; Elvis 2000; Baum \& McCarthy 2000; de Kool et al. 2001; Cecil et al. 2001; Levenson et al. 2001; Ruiz et al. 2001; Kaspi et al. 2002; Walter et al. 2002; Pounds et al. 2003b. a; Biller et al. 2004; Whittle \& Wilson 2004; Steenbrugge et al. 
2005; Rupke et al. 2005; Veilleux et al. 2005; Green 2006; Rice et al. 2006; Gabel et al. 2006; Krongold et al. 2007; McKernan et al. 2007).

In addition, although the exact normalization and scatter may evolve with redshift (potentially related to evolution in host galaxy properties setting the central potential, or evolution in feedback physics; see e.g. Croton 2006; Hopkins et al. 2007a), observations increasingly indicate that $\mathrm{BH}$-host correlations are in place even at high redshifts (Shields et al. 2003; Walter et al. 2004; Merloni et al. 2004; Adelberger \& Steidel 2005; Woo et al. 2006; Peng et al. 2006; Shankar et al. 2008) and in irregular or disturbed systems (Borys et al. 2005; Alexander et al. 2008; Kim et al. 2008), further demanding some form of self-regulation (as opposed to e.g. coincidence or fine-tuning from non selfregulating $\mathrm{BH}$ growth mechanisms).

If BHs grow in a self-regulated manner, then their evolution must be thought of as fundamentally determined by the growth of their host spheroid. If the spheroid is not large enough - if the central potential is no deeper - then a system that has already regulated its growth will simply enter into a high-feedback mode and immediately self-regulate when new gas is channeled into the central regions, regardless of how large a gas supply is fed to the BH. If this were not the case, again the problem of explaining the observed tightness of the BH-host correlations would present itself. "Growing the monster" requires not just a fuel supply, but also concomitant bulge growth, in order to raise the limit to which the $\mathrm{BH}$ can accrete before self-regulating.

Given the frequency of e.g. minor mergers and other processes that can remove angular momentum from at least some of the gas in the central regions of a galaxy (Lin et al. 2004; Woods et al. 2006; Maller et al. 2006; Barton et al. 2007; Woods \& Geller 2007; Stewart et al. 2007; Fakhouri \& Ma 2008; Younger et al. 2008; Hopkins et al. 2008d), simple cosmological calculations (as well as a survey of the abundance of massive bulges in the Universe) suggest that the bigger problem which must be overcome in order to allow $\mathrm{BH}$ growth may be the growth of bulges, not the removal of angular momentum from gas. In any event, the two cannot be considered separately.

This consideration leads to a new framework in which Seyfert and quasar fueling mechanisms must be evaluated. It has long been pointed out that major, gas-rich galaxy-galaxy mergers represent a viable means to fuel at least some quasars (Stockton 1978; Heckman et al. 1984; Sanders et al. 1988a, b; Stockton \& MacKenty 1987; Stockton \& Ridgwav 1991; Hutchings \& Neff 1992; $\quad$ Sanders \& Mirabel 1996; Bahcall et al. 1997; Canalizo \& Stockton 2001; Hutchings et al. 2003; Guyon et al. 2006; Dasyra et al. 2007; Bennert et al. 2008), and indeed it is generally accepted that although all quasars may not be mergers, all gas-rich mergers will induce AGN activity at some level (see references above and e.g. Hopkins et al. 2006). These encounters also have the advantage that, when two spirals merge, the disks are converted into spheroid and the bulge further grows via centrally concentrated star formation in a merger-induced starburst (Kormendy \& Sanders 1992; Mihos \& Hernquist 1994a b, 1996; Hibbard \& Yun 1999; Robertson et al. 2006b; Naab et al. 2006; Cox et al. 2006a; Hopkins et al. 2008e a. i. b). This deepens the central potential considerably - so not only does such a merger strip gas of angular momentum at all levels and provide a fuel source to the $\mathrm{BH}$, but it also increases the binding energy of the inflowing material, meaning that the $\mathrm{BH}$ must grow larger by a significant factor in order to self-regulate and ultimately lie on the observed BH-host correlations.

There are, however, many alternative mechanisms by which a nuclear BH could be fueled, and these will generically be more common in gas-rich (especially low-mass) galaxies. These include minor mergers (mass ratios $\sim 1: 10$ or so), angular momentum loss from stellar+gas bar systems in disks (for a review see Jogee 2004), the stochastic accretion of cold molecular clouds that happen to be near the BH or on scattered trajectories (Hopkins \& Hernquist 2006), and Bondi-Hoyle spherical accretion of hot gas from the diffuse, pressure-supported atmosphere in the central bulge (see e.g. Allen et al. 2006; Best et al. 2007, and references therein). However, these mechanisms are much less efficient (or even completely inefficient) at growing the bulge. Although it is possible to get some growth of the bulge and central potential from e.g. bars in isolated galaxies, most numerical experiments and cosmological simulations (Combes et al. 1990; Naab \& Burkert 2003; Bournaud et al. 2005; Athanassoula 2005; Debattista et al. 2006; Naab et al. 2007; Younger et al. 2008; Hopkins et al. 2008c d) as well as observations (O'Neill \& Dubinski||2003; Sheth et al.|2003; Jogee et al. 2004; Driver et al. 2007; Marinova \& Jogee 2007; Barazza et al. 2008) suggest that it is becomes difficult to get significant growth in these channels above a stellar mass $\sim 10^{10} M_{\odot}$, corresponding to a hosted $\mathrm{BH}$ mass $\sim 10^{7} M_{\odot}$, and the more massive bulge population will be increasingly dominated by systems whose growth primarily came in major mergers (for more detailed discussion, see Kormendy \& Kennicutt 2004; Maller et al. 2006; Hopkins et al. 2008c). This BH mass of $\sim 10^{7} M_{\odot}$ corresponds to a maximum bolometric luminosity of $\sim 0.3 \times$ $10^{12} L_{\odot}\left(1.3 \times 10^{45} \mathrm{erg} \mathrm{s}^{-1}\right)$ when radiating at the Eddington limit $^{2}$

Moreover, these distinctions are reinforced by a simple scaling: the minimum bolometric luminosity associated with "true" quasars is $\sim 10^{12} L_{\odot}$ (Soifer et al. 1987) (this translates to a typical optical magnitude $M_{\mathrm{B}}<-23$; the traditional Seyfert/quasar division introduced by Schmidt \& Green (1983) as an observational, not physical division). Fueling a Seyfert $\left(L_{\mathrm{bol}} \leq 10^{12} L_{\odot}\right)$ for a Salpeter (1964) time $t_{S}=$ $4.2 \times 10^{7} \mathrm{yr}$ (the $e$-folding time for exponential growth at the Eddington limit, given a typical radiative efficiency expected of BH accretion $\left.\epsilon_{r} \equiv L_{\mathrm{bol}} /\left(\dot{M} c^{2}\right) \sim 0.1\right)$, similar to the lifetime of a high-accretion rate episode suggested by various observational constraints (clustering, sizes of radio jets, and the transverse proximity effect; for a review see Martini 2004), requires $\lesssim 10^{7} M_{\odot}$ worth of accreted material (unsurprising, given that this luminosity corresponds to a $\mathrm{BH}$ of around this mass at its Eddington limit). Allowing for some reasonable efficiency of fueling (say $\sim 10 \%$ of the mass within the $\mathrm{BH}$ radius of influence or vicinity of the $\mathrm{BH}$ ) this is still comparable to the mass in a single or a few giant molecular clouds $\left(\sim 10^{7}-10^{8} M_{\odot}\right)$. The limits become less stringent as one considers lower luminosities (more typical Seyferts with $L_{\text {bol }} \sim 10^{11} L_{\odot}$ requiring only $\sim 10^{6}-10^{7} M_{\odot}$ worth of gas). There are many processes that could sufficiently disturb the gas supply in the central regions of the galaxy so as to produce such an event - for example, even in the Milky Way, a

\footnotetext{
2 The Eddington limit giving $L_{\mathrm{bol}}=3.3 \times 10^{4} L_{\odot}\left(M_{\mathrm{BH}} / M_{\odot}\right)=1.29 \times$ $10^{38} \operatorname{erg~s}^{-1}\left(M_{\mathrm{BH}} / M_{\odot}\right)$.
} 
sufficient random perturbation (with mass of this magnitude) - a near pass to the BH of another giant molecular cloud complex or massive star cluster - could torque such a mass (already near the $\mathrm{BH}$ in molecular clouds) into accretion (see e.g. Genzel et al. 1994, and references therein). Given the relative ease of triggering and ubiquity of such systems, there is no reason to invoke a rare and violent mechanism such as major mergers - in fact, the natural expectation is that more mundane, far more frequent processes should dominate.

However, fueling a bright quasar (say $L_{\text {bol }} \sim 10^{14} L_{\odot}$ for $\left.M_{B} \approx-28\right)$ for a similar duration requires $\gtrsim 10^{9}-10^{10} M_{\odot}$ in gas - i.e. channeling an entire typical galaxy's supply of gas onto the $\mathrm{BH}$ itself. Given the efficiency of star formation when dense gas is accumulated in the central regions of a galaxy, almost any reasonable model demands more like $\sim 10^{11} M_{\odot}$ worth of gas within the central $\sim 50-100 \mathrm{pc}$ (even for objects that are fainter, but still quasars, at $L_{\mathrm{bol}} \gg$ $\left.10^{12} L_{\odot}\right)$. Given that the timescale needed is comparable to or shorter than the dynamical time of the galaxy itself, a massive, galaxy-wide perturbation is needed (essentially the entire supply of galactic gas must be stripped of angular momentum and free-fall to the center in less than a single rotational period of the disk), and the only mechanism expected in standard cosmologies to be capable (let alone expected) to produce such a perturbation is a major merger (any disk instability or minor merger, by the nature of the amplification of that instability or dynamical friction, respectively, could neither channel the required mass in an absolute sense nor do so in less than a few disk orbital periods).

This leads to the idea, motivated by detailed models for accretion in mergers and isolated systems, that there is some characteristic host bulge mass/BH mass and corresponding quasar luminosity (which happens to correspond to the observationally defined Seyfert-quasar division) below which these more ubiquitous mechanisms dominate AGN fueling (being more common and requiring less bulge growth to deepen the central potential in this mass regime). Above this division, less violent mechanisms are simply inefficient (they may still happen, but they do not sufficiently raise the bulge mass, so BHs quickly self-regulate and do not experience any significant lifetime of high-Eddington ratio growth) and the population requires more extreme mechanisms such as major mergers to build the most massive bulges and (corresponding) BHs.

Here, we outline observational constraints that can be used to test this idea, and provide constraints on where non-major merger related fueling mechanisms do or do not dominate the $\mathrm{BH}$ and AGN populations. These various accretion processes have different observational signatures, particularly when we recognize that, in a self-regulated growth scenario, they must be treated not just as $\mathrm{BH}$ growth mechanisms but simultaneously grow bulges. In $\S 2,6$ below, we outline a few such tests and illustrate some preliminary constraints from previous works, and in $\S 7$ we summarize the results.

For ease of comparison, we convert all observations to bolometric luminosities given the appropriate bolometric corrections from Hopkins et al. (2007d) (see also Elvis et al. 1994; Richards et al. 2006). We adopt a $\Omega_{\mathrm{M}}=0.3, \Omega_{\Lambda}=0.7, H_{0}=$ $70 \mathrm{~km} \mathrm{~s}^{-1} \mathrm{Mpc}^{-1}$ cosmology and a Salpeter (1955) stellar initial mass function (IMF), and normalize all observations and models appropriately (note that this generally affects only the exact normalization of quantities here, not the qualitative conclusions). All magnitudes are in the Vega system.

\section{HOST GALAXY MORPHOLOGIES}

A great deal of attention has been paid to the issue of testing merger-induced quasar fueling models by looking for signatures of disturbance in e.g. quasar and AGN hosts (Bahcall et al. 1997; Canalizo \& Stockton 2001; Floyd et al. 2004; Zakamska et al. 2006; Pierce et al. 2007). However, this is extremely difficult, especially at the high redshifts and luminosities of interest. where this fueling mechanism is expected to dominate. Moreover, models predict that the quasar phase in mergers occurs at the end of the merger, in the background of a largely relaxed remnant (Di Matteo et al. 2005; Hopkins et al. 2005d a, 2006), and at these times experiments with e.g. automated morphological classification systems such as Gini-M20 and CAS (Lotz et al.2008a) as well as optical identifications (Krause et al. 2008) suggest that even with perfect image depth, merger induced-quasar hosts would be uniformly classified as relaxed, non-merging systems. There is some prospect for deeper observations to reveal new insights, as e.g. very nearby systems (previously classified as relaxed) have, upon deeper imaging (Bennert et al. 2008) been revealed as clearly interacting, and imaging of redder or more obscured quasars has identified interaction-dominated populations (Hutchings et al. 2003, 2006; Kawakatu et al. 2006; Urrutia et al. 2008), with degree of interaction increasing in more infrared-luminous populations as well (Guyon et al. 2006), but this remains prohibitively expensive for most systems of interest.

However, we highlight a more mundane but equally constraining point here. Viewed during the final stages of a major merger, it may be impossible to (with present observations) say whether there was a recent merger/disturbance, but it is possible to identify whether the hosts are spheroids or disks. The hosts being spheroids does not uniquely imply a merger origin of the hosted quasars, of course, but their being disks would clearly put strong constraints on merger mechanisms ${ }^{3}$.

Indeed, a number of observational studies have pointed out that, among the high Eddington-ratio population (important to distinguish from possible low-Eddington ratio, non thindisk accretion in old systems), there is a characteristic difference roughly above and below the Seyfert-quasar divide. Broad-line Seyferts tend to live in disk-dominated host galaxies: clearly, most of these systems are not recent major merger remnants. Broad-line quasars, on the other hand, have predominately elliptical hosts, candidates for recent mergers. In fact, Dunlop et al. (2003); Floyd et al. (2004) find that the transition regime is quite narrow - around a factor of a few in luminosity, where the population goes from strongly disk to bulge dominated.

Figure 1 presents the results of studies that compare the morphological distribution of low and high-luminosity AGN selected in the same manner. We show the luminosity below which the AGN sample is dominated by either disks or an apparently random mix of disks and ellipticals (i.e. the sample shows no strong preference for early-type hosts) and above which the sample is dominated by ellipticals, from the PG quasar sample (Dunlop et al. 2003), the sample of Type

${ }^{3}$ Disks can survive mergers Barnes \& Hernquist (1996); Barnes (2002); Springel \& Hernquist (2005); Robertson et al. (2006a); Hopkins et al. (2008d h); but for major mergers with large bulge growth (the cases of interest here), the remnant disks are generally small and compact and do not dominate the stellar mass. Large disks in the remnant require special conditions (usually not being coupled to substantial $\mathrm{BH}$ fueling); so observations of disk-dominated AGN hosts would still present strong constraints on what, if any, contribution to fueling comes from major mergers. 

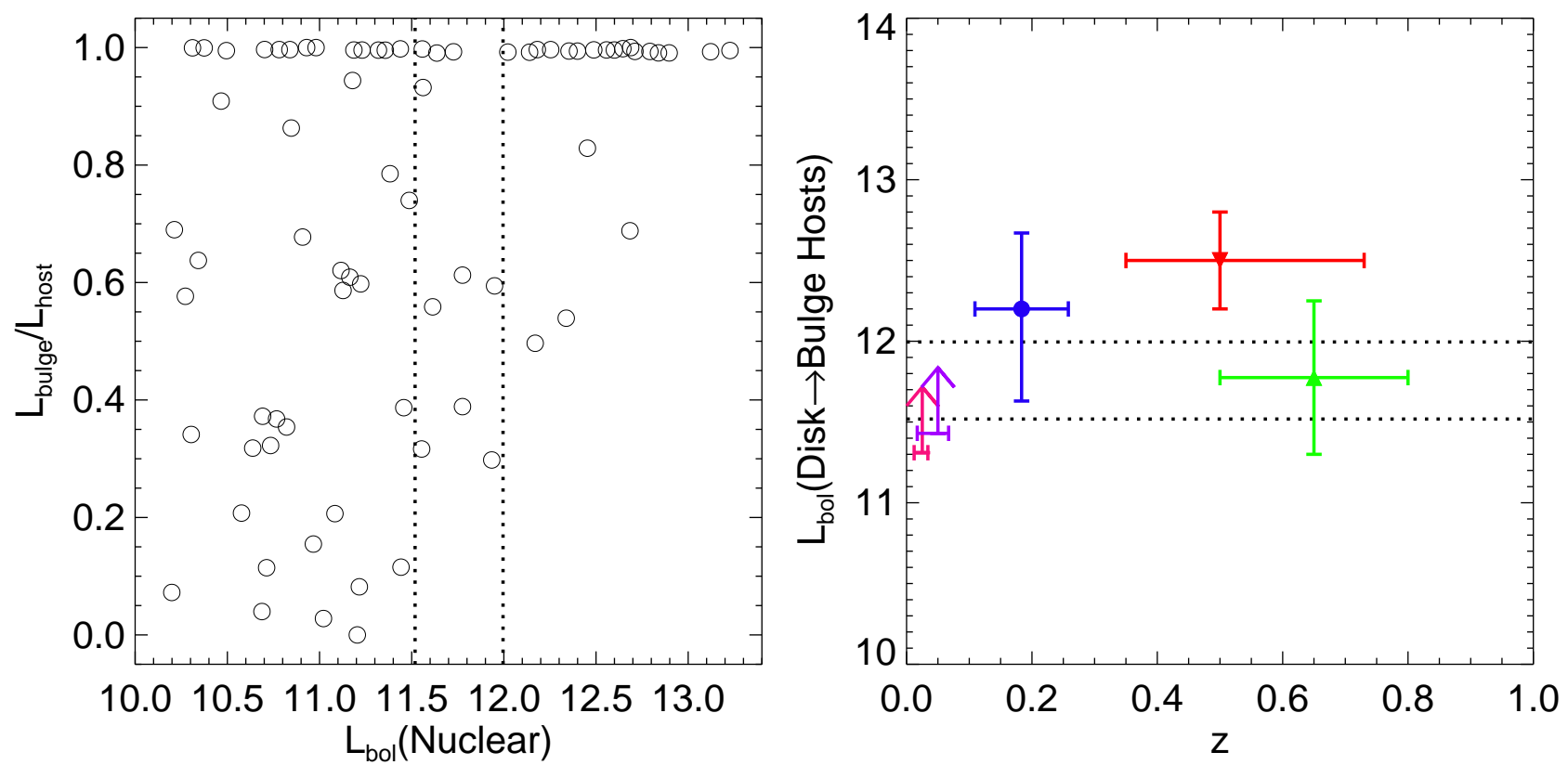

FIG. 1. - Left: Host galaxy morphology (bulge-to-total luminosity ratio) versus AGN bolometric luminosity, from the sample of Dunlop et al. (2003). Around the characteristic Seyfert-quasar divide $\left(L_{\mathrm{bol}} \sim 10^{12} L_{\odot}\right)$, hosts transition from being a representative mix of bulges and disks to being exclusively bulgedominated (Es and SOs): major-merger remnants must be bulge dominated (even if tidal disturbances fade rapidly), whereas non-merger fueling mechanisms allow a random mix of non-spheroid hosts. Right: Luminosity where the observed AGN host galaxy populations transition from being essentially random or disk-dominated (at low- $L$ ) to bulge-dominated (at high- $L$ ), from the observed samples of Malkan et al. (1998, pink arrow), Kauffmann et al. (2003, purple arrow), Dunlop et al. (2003, blue circle), Zakamska et al. (2006, 2008, red inverted triangle), and Rigby et al. (2006, green triangle). The low- $z$ samples do not include bright quasars or see such a transition, so set only a lower limit here. At redshifts $z \lesssim 1$ where morphologies are observed over the dynamic range of interest, a characteristic transition in host properties appears roughly at similar luminosity.

II quasar hosts in Zakamska et al. (2006, 2008), and similar X-ray selected Type II samples (Rigby et al. 2006). Despite significant differences in selection and many of the observed properties of the quasars in these samples, the results are similar in each study: a sharp transition (over a factor $\sim 2$ in $L$ ) between disk/star-forming (or random) host galaxies at $L_{\text {bol }} \lesssim 10^{12} L_{\odot}$ and predominantly elliptical or bulgedominated hosts at brighter luminosities.

\section{RELIC BULGE MORPHOLOGIES/PHENOTYPES}

Numerical simulations and, increasingly, observations have established that at least major mergers form "classical bulges," " whose properties resemble those of scaled-down ellipticals (see references in $\$ 1$. On the other hand, the alternative fueling mechanisms discussed in $\$ 1$ (bars and sufficiently minor mergers), to the extent that they result in any bulge formation at all, yield "pseudobulges," with distinct properties: lower Sersic indices and concentrations more analogous to the progenitor disk, larger sizes, a higher degree of rotational support, more ongoing star formation and bluer colors, and often a boxy or "peanut"-shaped morphology or clear inner disk. If there is a characteristic transition regime above and below which certain processes dominate, this should be imprinted in the detailed classes of bulges left as relics.

Figure 2 compares the mass function of BHs in local bulges, separated into classical and pseudobulges. Given the mass

\footnotetext{
${ }^{4}$ The consequences of minor mergers are more ambiguous. Most minormerger remnants would be classified as "classical" bulges down to mergers of mass ratios $\sim 8: 1$ or so, but results are not clear for more minor interactions. In minor mergers, the dependence of the remnant structure on the initial orbital parameters and other properties increases - for a large range in such parameters, the combination of tidal stripping and large orbital times makes it ambiguous whether or not the system can even be classified as a genuine "merger."
}

function of bulges/spheroids, we convolve with the observed BH-bulge mass relation (Marconi \& Hunt 2003) and its scatter to obtain the corresponding $\mathrm{BH}$ mass function. Note that although there may be an offset between the BH-host galaxy correlations in pseudo and classical bulges, suggested by theory (Younger et al. 2008) and observations ( $\mathrm{Hu}$ 2008; Greene et al. 2008; Gadotti \& Kauffmann 2008), the magnitude of this offset is sufficiently small that it makes no difference to our comparisons here ${ }^{5}$.

Given the spheroid mass function, we classify systems as pseudo or classical bulges given one of three different (commonly adopted) observational proxies. First, their fitted Sersic indices (systems with $n_{s}<2$ being predominantly pseudobulges, systems with higher $n_{s}$, similar to observed ellipticals, being classical bulges; see Fisher \& Drory 2008); we adopt the bivariate mass-Sersic index distribution from Driver et al. (2006); Graham et al. (2007). Second, their colors (bluerthan-average systems being predominantly pseudobulges); Drory \& Fisher (2007) and Fisher (2006) demonstrate a tight correlation of this nature and we adopt the corresponding color divisions from Driver et al. (2007). Third, their host galaxy types (bulges in systems later than Sbc being predominantly pseudobulges), with the type-separated mass functions from Kochanek et al. (2001) and type-dependent $B / T$ values from Aller \& Richstone (2002); Balcells et al. (2007). Regardless of the definition, the estimators agree on a similar mass $\sim 1-3 \times 10^{7} M_{\odot}$ where the transition occurs, which at the Eddington limit gives a luminosity $\sim 10^{12} L_{\odot}$ corresponding to the disk-spheroid host division above.

\section{SMALL-SCALE CLUSTERING}

\footnotetext{
${ }^{5}$ If adopted, this shifts the mass where the bulge/BH population transitions from pseudobulge to classical bulge-dominated to lower mass by a factor $\sim 1.5-2$, comparable to the existing systematic uncertainties.
} 


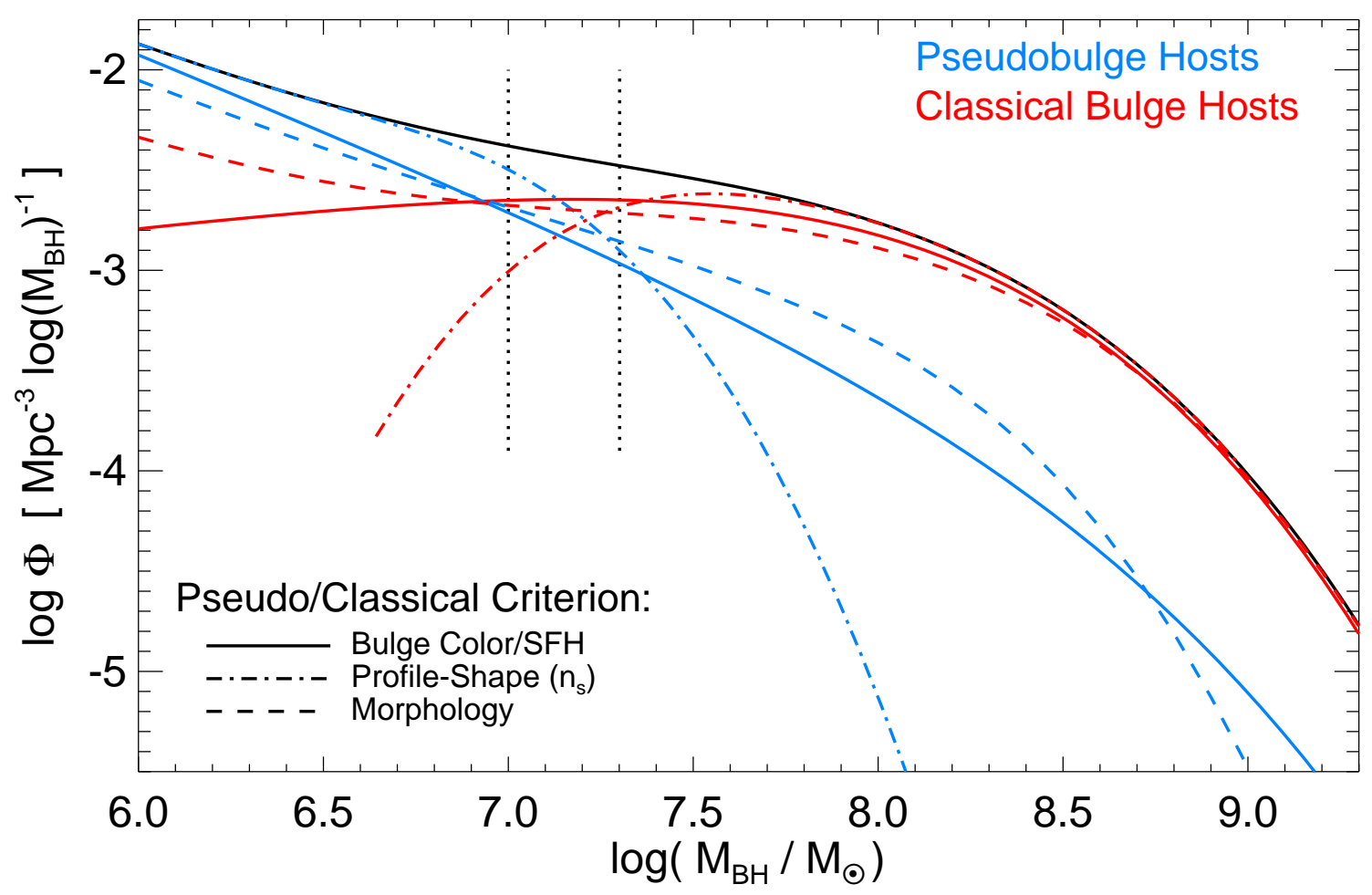

FIG. 2.- Local BH mass function (black), divided into BHs in "pseudobulges" (blue; generally formed in non-merger related secular evolution) and "classical" bulges (red; formed in mergers). Different lines adopt different observational criteria for dividing the observed bulge population into pseudo and classical bulges (details in text). Regardless, a characteristic transition occurs at a BH mass $\sim 1-3 \times 10^{7} M_{\odot}$, which at the Eddington limit corresponds to the same $\sim 10^{12} L_{\odot}$ luminosity in Figure 1 The BH mass function is consistent with merger-induced fueling above this mass/luminosity limit, and non-merger fueling below the limit.

The merger rate is enhanced in small-scale overdensities, and this means that (with respect to a random, nonmerger population with similar masses and large-scale clustering properties) recent merger remnants will exhibit enhanced clustering on small scales (within of order the size of a host halo, $\sim 200 \mathrm{kpc}$ ). This is seen observationally in recent merger remnants and post-starburst ellipticals (Goto 2005), and both cosmological simulations and analytic models (Scannapieco \& Oh 2004; Thacker et al. 2006; Hopkins et al. 2007c, 2008g) predict that it should leave an observable signature in the small-scale clustering of quasars, to the extent that they are a merger-induced population.

Indeed, such a small-scale excess has been seen (Hennawi et al. 2006), in both low-redshift (Serber et al. 2006) and high-redshift (Myers et al. 2007) quasar populations. Interestingly, however, Serber et al. (2006) see no such excess in the low-redshift Seyfert population, with a transition luminosity (between the presence and absence of such a feature) corresponding closely to that in Figure 1

We summarize these results in Figure 3. At low redshift, we compare the relative small-scale bias (amplitude of the observationally estimated bias at $r$ relative to that as $r \rightarrow \infty$ ) for Seyferts and quasars in the Serber et al. (2006) sample (see also Padmanabhan et al. 2008, who more robustly describe this difference as requiring that quasars have a higher halo occupation in small groups). The excess seen for quasars (and not for Seyferts) is very similar (both qualitatively and quantitatively in magnitude and characteristic $r$ where the effect begins) to that seen in known recent merger remnants and post-starburst ellipticals (Goto 2005) and predicted for recent galaxy merger remnants (Thacker et al. 2006; Hopkins et al. $2007 \mathrm{c}, 2008 \mathrm{~g}$, c).

We can summarize this result by comparing the average bias/clustering amplitude within $\sim 100 \mathrm{pc}$, relative to that at large $r$ (i.e. the "excess" bias on one-halo/group scales), for different samples as a function of redshift. Quasar observations extend this (albeit with increasing error bars) from $z=0-2.5$, consistent with a relatively constant small-scale clustering excess of a factor $\sim 2$. Unfortunately, comparable observations for Seyferts exist only for the low-redshift $(z \lesssim 0.5)$ sample in Serber et al. (2006). This sample is consistent with no small-scale excess (i.e. requiring that Seyferts show no preference for groups or dense small-scale environments, relative to a random galaxy in a halo of similar mass), or at least any such excess is smaller than that in quasars at high significance, but observations at lower luminosities are required to extend this to higher redshifts.

\section{REDSHIFT EVOLUTION}

The rates and fractions of gas-rich mergers are expected and observed to increase at higher redshift (albeit with debate regarding how rapid this increase may be; see e.g. Patton et al. 2002; Conselice et al. 2003; Bundy et al. 2004; Lin et al. 2004; Xu et al. 2004; De Propris et al. 2005; Cassata et al. 2005; Wolf et al. 2005; Bundy et al. 2005; Lotz et al. 2006, 2008b; Bell et al. 2006; Bridge et al. 2007; Kartaltepe et al. 2007). A truly random subpopulation, on the other hand, will of course have a constant fraction (even a gas-fraction dependent population driven by e.g. random triggering through encounters with molecular clouds in a gas-rich disk will show similar behavior; Hopkins \& Hernquist 2006), and observa- 

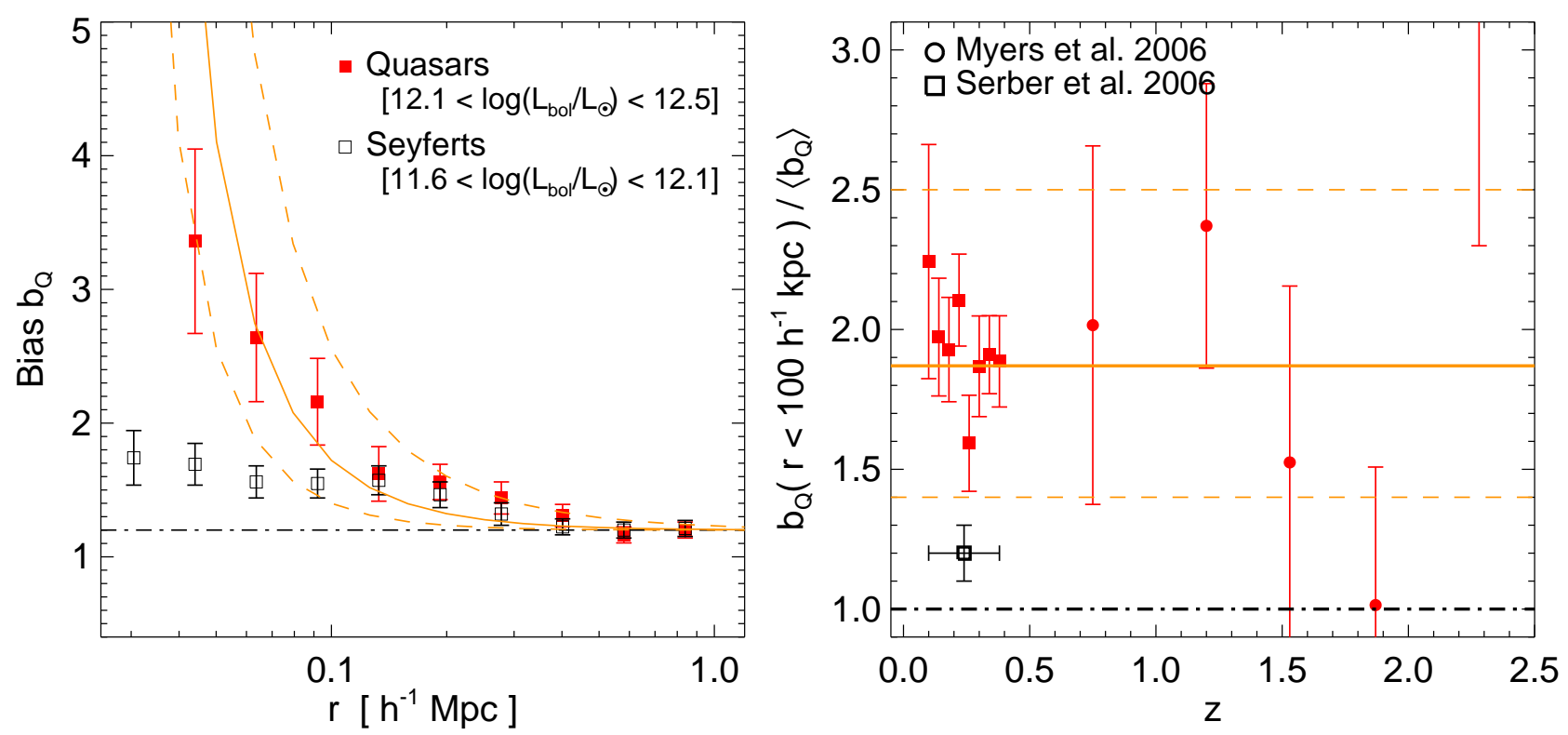

FIG. 3.- Excess small-scale clustering of quasars and Seyferts. Left: Observed correlation functions from Serber et al. (2006) for optical quasars (red filled squares) and Seyferts (black open squares) - we plot the bias as a function of scale radius determined from the correlation function at each radius relative to that as $r \rightarrow \infty$ (i.e. dividing out the best-fit large-scale dark matter correlation function). Dot-dashed line shows a constant bias as a function of scale (i.e. no excess of small-scale densities, expected if the galaxies are a random subpopulation with no preference for small groups), solid (dashed) orange line shows the predicted excess on small scales expected for recent merger populations from cosmological models and simulations (reflecting a preference for small group environments; see Thacker et al. 2006; Hopkins et al.2008g). Right: Ratio of the mean bias at small radii $\left(r<100 h^{-1} \mathrm{kpc}\right)$ to that at large radii (the asymptotic values in the left panel), at all redshifts where this has been observed. Lines show the predicted excess from the previous panel. We show the same observations from|Serber et al. (2006) (squares), as well as high-redshift observations from Myers et al. (2007) (circles), which include only quasars. Quasars display a similar excess at low and high redshifts (consistent with a constant excess at all $z$ ), expected in mergers. Seyferts show no such excess at low redshifts, but high- $z$ observations are needed.

tions suggest the bar fraction in disks is constant (or even decreasing) with redshift (Sheth et al. 2003; Jogee et al. 2004). Likewise, the fraction of galaxies experiencing minor mergers (mass ratios $\gtrsim 1: 10$ ) is essentially constant at $\sim 1$ at all redshifts (not surprising given that the dynamical friction time for such a merger is of order the Hubble time; Boylan-Kolchin et al. 2008).

If the average quasar/Seyfert lifetime is (at least in a statistical sense) some constant duration or approximately constant fraction of the observable merger lifetime - as suggested by simulations (Hopkins et al. 2005d c. b), mock observations (Lotz et al.2008a; Krause et al. 2008), and observational constraints on quasar lifetimes and duty cycles (see e.g. Martini 2004, and references therein) - then this may be reflected in the evolution of the number density or fraction of halos hosting AGN of different luminosities (albeit with the caveats in $\S 7$.

Indeed, it is well-established that the number density of bright quasars increases rapidly with redshift, peaking at $z \sim$ $2-4$ (above which the population must decline, as the absolute number of intermediate to high mass halos/galaxies that could host such massive BHs rapidly decreases), whereas the number density of low-luminosity Seyferts remains relatively constant, peaking at very low redshifts and declining above $z \sim 0.5-1$.

Figure 4 summarizes these results and compares them to expectations of merger rates. First, we compare the absolute number density of quasars above the luminosity threshold of interest $\left(L_{\text {bol }}>10^{12} L_{\odot}\right)$ and Seyferts $\left(10^{9} L_{\odot}<L_{\text {bol }}<\right.$ $\left.10^{12} L_{\odot}\right)$. We compare to the number density of observed mergers $^{6}$ (with masses $\sim 10^{10}-10^{11} M_{\odot}$ such that they are

\footnotetext{
6 Technically, we are interested in the number of gas-rich mergers, which many of the observations considered do not specifically distinguish. How-
}

representative of the galaxy masses hosting these BHs and AGN). To lowest order, the number density of merger-induced quasars will scale as the merger number density times the ratio of their observable lifetimes, $t_{Q} / t_{\text {merger. }}$. Observational selection of mergers is sensitive to the full time of orbital decay from some radius (in the case of pair selection) or time since first passage (when morphological disturbance is excited) until some number of dynamical times after the mergers when features relax; calibration of these selection methods against high-resolution hydrodynamic merger simulations suggests an observable lifetime $t_{\text {merger }} \sim 1-2 \mathrm{Gyr}$. On the other hand, while the total time of activity at lower levels may be much longer, a typical bright quasar phase lasts only $\sim 20-40 \mathrm{Myr}$ (see references above or Martini 2004, or considering the time for e.g. doubling of $\mathrm{BH}$ mass in a high Eddington ratio phase). This suggests a value of $t_{Q} / t_{\text {merger }} \sim 1 / 25-1 / 50$, which we find provides a good match to the relative number densities of quasars and mergers (but in any case we are not trying to compare the absolute number of objects, but their relative redshift evolution; for our purposes the normalization is arbitrary).

We also compare with the prediction from cosmological models: first, the dark-matter only merger fraction (adopting the observable merger timescales from Boylan-Kolchin et al. (2008); Hopkins et al. (2008g) and halo merger rates from Fakhouri \& Ma (2008)), for halos sufficiently massive to host such a quasar (using the Eddington limit as a lower bound and assuming a fixed $M_{\mathrm{BH}}-M_{\text {halo }}$ relation from da Angela et al. 2008 , this gives $M_{\text {halo }} \gtrsim 10^{11.5} h^{-1} M_{\odot}$; although the predic-

ever, they are most sensitive to these mergers (e.g. spiral-spiral mergers), as they are both brighter and tend to excite more dramatic tidal features and morphological disturbance than e.g. a spheroid-spheroid merger of the same mass. Moreover, where observations separate the two, the number of "dry" or dissipationless spheroid-spheroid mergers is much smaller $(\sim 10 \%)$ than the number of gas-rich mergers (e.g. Lin et al. 2008). 

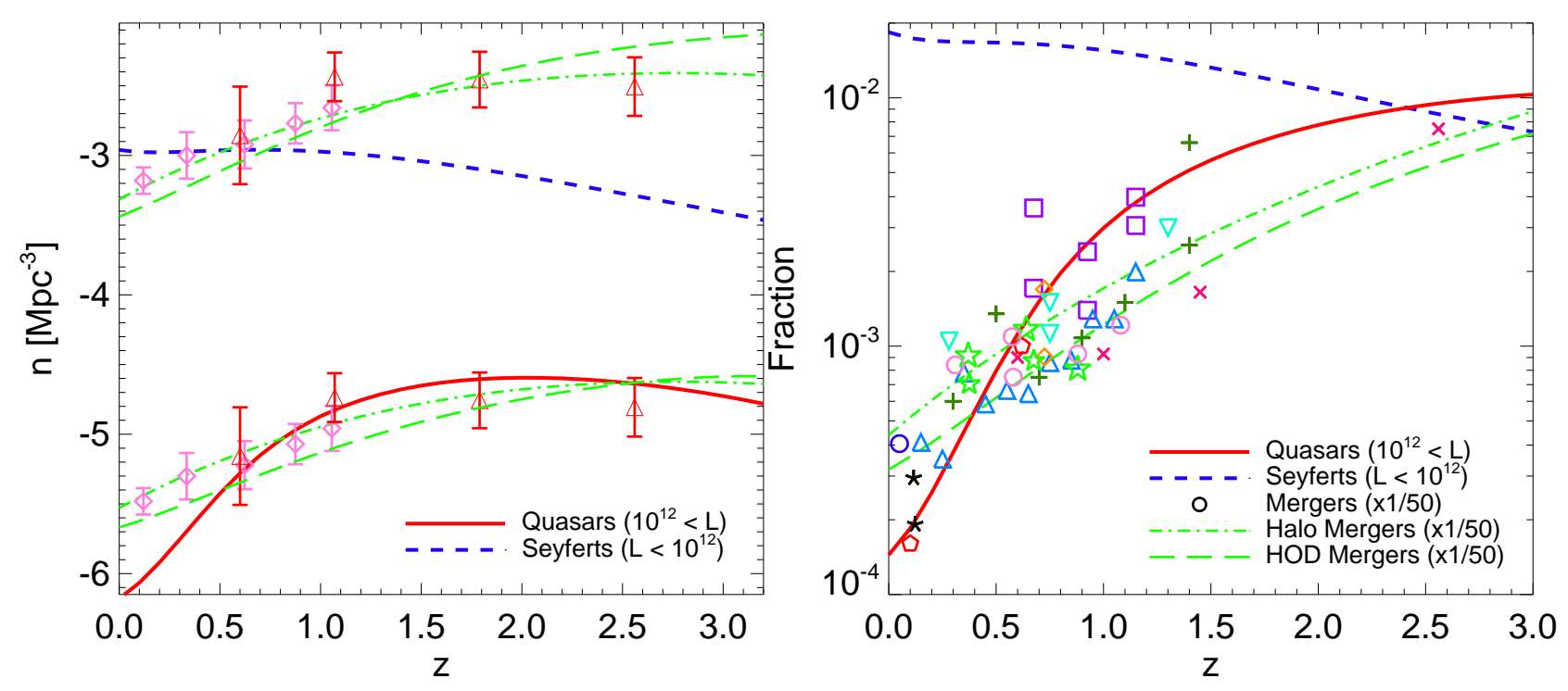

FIG. 4.- Left: Observed quasar (red solid) and Seyfert (blue dashed) number densities as a function of redshift, from the compilation of AGN luminosity functions in Hopkins et al. (2007d) (see also Hasinger et al. 2005; ; Shankar et al. 2007, and references therein). We compare with the number density of gas-rich (disk-disk) mergers observed (Lin et al. 2008; Conselice 2006, pink diamonds and magenta triangles with dotted lines, respectively), multiplied by 1 (upper) and $1 / 50$ (lower), reflecting e.g. an approximate ratio of the visible merger timescale ( $\gtrsim \mathrm{Gyr}$ ) to the quasar/Seyfert lifetime (we are not attempting to constrain the lifetimes here, only the relative redshift evolution). We also compare with predicted merger rates in corresponding dark matter halos (sufficiently massive to host each population; see Fakhouri \& Ma 2008, green dot-dashed) and halo occupation models of galaxy-galaxy mergers (also with corresponding mass limits; Hopkins et al. 2008g, green dashed). Right: Same, in terms of the quasar/Seyfert/merger fraction. AGN fractions are defined in terms of halo occupation models (see text) or the minimum galaxy/BH mass required to host a given AGN. We add merger fractions from Conselice et al. (2003, magenta $\times$ 's), Bundy et al. (2004, 2005, green stars), Lin et al. (2004, pink circles), Xu et al. (2004, dark blue circles), De Propris et al. (2005, black asterisks), Cassata et al. (2005, cyan inverted triangles), Wolf et al. (2005, orange diamonds), Lotz et al. (2006, 2008b, dark green +'s), Bell et al. (2006, red pentagons), Bridge et al. (2007, violet squares), and Kartaltepe et al. (2007, blue triangles). Quasar number densities/fractions rise with redshift and peak/flatten at $z>2$, in agreement with merger rates, whereas Seyfert number densities are constant or decline at increasing redshift, more similar to a population with random (constant in redshift) triggering or the observed bar population (Sheth et al. 2003; Jogee et al. 2004).

tions are not sensitive to halo mass). Second, the predicted galaxy merger rate from halo occupation models, fixed to reproduce the galaxy mass functions, clustering, and their evolution (here from Hopkins et al. 2008c). Both are rescaled by the same approximate ratio of lifetimes.

We can reverse this comparison and show the same trends in terms of the "active fraction" - i.e. the fraction of available halos (above a given halo mass or hosting a given $\mathrm{BH}$ mass) that host a quasar or AGN within some luminosity range. This is just the observed merger fraction multiplied by the ratio of lifetimes $t_{Q} / t_{\text {merger }}$. The quasar fraction in terms of e.g. halo mass can be defined in detail using halo occupation models for quasar clustering (Croom et al. 2005; Porciani \& Norberg 2006; Fine et al. 2006; Shen et al. 2007; da Angela et al. 2008) or simply taking the ratio of the number of quasars to the number density of halos sufficiently massive to host any QSO in the relevant luminosity bin (both results are identical for our purposes). Clearly, regardless of the details of the observable lifetimes, the quasar number density and fraction rises with redshift in a manner roughly consistent with the predicted and observed rates of mergers (somewhat faster, in fact, but we have ignored subtleties here such as evolution in quasar lifetimes, dependence on host properties, and dependence on the overall gas supply and gas content of the merger progenitors).

We repeat these experiments for the Seyfert population (in this case rescaling by a ratio of lifetimes $\left.t_{\text {Seyfert }} / t_{\text {merger }} \sim 1\right)^{7}$.

\footnotetext{
${ }^{7}$ Note that we are here considering all Seyferts down to very low AGN luminosities $L_{\text {bol }} \sim 10^{9} M_{\odot}\left(M_{B}=-15\right.$ or $L_{2-10 \mathrm{keV}} \sim 10^{41.5} \mathrm{erg} \mathrm{s}^{-1}$; including obscured sources at these luminosities) - at these luminosities expected
}

These trends, regardless of normalization, cannot match the observed redshift dependence of Seyfert number density or fractions. On the other hand, the weakly evolving (or even decreasing with redshift) observed bar fractions can provide a very plausible match, given an average ratio of lifetimes $\sim 0.3-2$, as can an essentially constant $\sim 1$ fraction of systems undergoing a minor merger, or the fraction of disks expected to be undergoing stochastic activity triggered by random crossings of molecular clouds with the $\mathrm{BH}$ radius of influence (see Hopkins \& Hernquist 2006).

\section{HOST GALAXY COLORS AND STAR FORMATION HISTORIES}

Finally, recent merger remnants should exhibit different host galaxy colors and star formation properties relative to a random sub-population of "normal" galaxies (or bar/minor merger remnants). These characteristics are difficult to measure in practice, especially since whatever merger-induced starburst occurs will generally leave a dissipative, compact stellar population (effective radii of the young populations often $\lesssim 1-2 \mathrm{kpc}$ in mergers and remnants; Scoville et al. 1986; Sargent et al. 1987; Kormendy \& Sanders 1992; Hibbard \& Yun 1999; Genzel et al. 2001; Tacconi et al. 2002; Rothberg \& Joseph 2004, 2006), which could be masked by the quasar PSF and will rarely dominate the bolometric light output of luminous QSOs.

That being said, there has been tremendous effort to constrain the stellar populations of AGN observed

lifetimes are large, $\sim$ Gyr (duty cycles are observationally seen to be $\sim 10 \%$ ), so this ratio $t_{\text {Seyfert }}\left(>10^{9} L_{\odot}\right) / t_{\text {merger }} \sim 1$ is expected. 
at many different luminosities and in different wavelengths (e.g. Brotherton et al. 1999; Canalizo \& Stockton 2001; Farrah et al. 2003, 2005; Kauffmann et al. 2003; Yip et al. 2004; Jahnke et al. 2004a. b; Sánchez et al. 2004; Vanden Berk et al. 2006; Barthel 2006; Zakamska et al. 2006). From these efforts, it has been established that AGN and star formation activity are broadly contemporaneous. This alone does not distinguish between different fueling models, so long as a reasonable gas supply is available. However, the manner in which the two accompany one another does distinguish between models.

A star-forming (disk) galaxy that experiences a minor (Seyfert-level) triggering event, be it a minor merger, bar or disk instability, or stochastic interaction of $\mathrm{BH}$ and giant molecular cloud, will continue (after the AGN episode) as just as much a star forming system as before. This is true even in the most extreme feedback models, as it is nearly impossible for an AGN episode to influence star formation in a dense, self-shielding galactic disk with a very large mass (see e.g. the simulations and analytic calculations in Murray et al. 2005; Thompson et al. 2005; Hopkins et al. 2008c). Moreover, it is physically not possible to channel the entire galactic gas supply into the center of the galaxy (to be consumed in a starburst) in such events (by definition not so in random events or minor mergers, and by various conservation laws in disk instabilities).

On the other hand, a major merger can efficiently exhaust the galactic gas supply by channeling gas into nuclear starbursts (Mihos \& Hernquist 1994b, 1996; Cox et al. 2006b, 2008), leading to associated star formation and AGN fueling with a long tail of AGN activity after the final coalescence and rapid decline in the star formation rate owing to exhaustion of the nuclear gas (Springel et al. 2005b.a). In short, much (although not all) of the merger-induced AGN activity will come after the system has already begun to redden as a poststarburst system.

This is supported by a large number of observations of stellar populations in AGN. High Eddington ratio systems are observed to be preferentially in bluer-than-average spheroids, but systems that are still redder than typical disks of the same mass - i.e. spheroid-dominated systems in the so-called "green valley"8 (see e.g. Brotherton et al. 1999; Kauffmann et al. 2003; Sánchez et al. 2004; Kewley et al. 2006; Nandra et al. 2007; (Silverman et al. 2008).

In particular, Vanden Berk et al. (2006) argue that brighter quasars (as opposed to Seyferts) preferentially show poststarburst activity (rather than a strong preference for large amounts of on-going star formation), as expected in efficient mergers (and opposite the expectation for secular fueling mechanisms), with the strength of these signatures increasing in more luminous systems. Shi et al. (2007) demonstrate that the recent star formation activity associated with Seyfert galaxies at low redshifts implies that - although there was some pre-Seyfert star formation - they were sub-LIRG systems at the peak of their pre-Seyfert star formation episode (even assuming this star formation came in a rapid burst, they would have been less luminous than $L \sim 10^{11} L_{\odot}$ ), typical of star-forming galaxies. In contrast, the recent star formation they identify in quasars is enhanced - if it came in a sudden

8 The region in the color-magnitude or color-mass diagram (see e.g. Strateva et al. 2001; Baldry et al. 2004) between the locus of star-forming galaxies (the "blue cloud") and passively evolving spheroids (the "red sequence"). pre-quasar burst, it would imply these systems were (briefly) LIRG/ULIRG systems $\left(L \gtrsim 10^{11} M_{\odot}\right)$, a luminosity regime that is empirically almost universally associated with mergers over this redshift range (Allen et al.|1985; Joseph \& Wright 1985; Armus et al. 1987; Sanders \& Mirabel 1996).

These luminosity thresholds may evolve with redshift, but as a qualitative statement the same should be true. At $z=2$, for example, Lutz et al. (2008) argue that the quasars in their sample require $\sim 1000 M_{\odot} \mathrm{yr}^{-1}$ recent star formation episodes (see also Wang et al. 2008), at the hyper-LIRG level demanding (in almost any model) and ubiquitously associated with major mergers, but find that their Seyferts $\left(L_{\text {bol }} \sim 3 \times 10^{11} L_{\odot}\right)$ reflect lower-level recent star formation (consistent with LIRG-like luminosities, which simulations and observations suggest may commonly arise from very gas-rich disks at these redshifts without major mergers; Le Floc'h et al. 2005; Reddy et al. 2006; Yan et al. 2007; Dasyra et al. 2008; Saijina et al. 2008).

It has been argued that quasars in general exhibit poststarburst signatures of a magnitude such that $\gtrsim 10 \%$ of the stellar mass must have been formed in a recent starburst (recently see e.g. Higdon et al. 2008, for a review see Canalizo \& Stockton 2001). Such a large mass fraction is difficult to explain through any fueling mechanism other than a major merger, and is also in very good agreement with the major-merger induced starburst masses implied by observations and simulation models of the the surface brightness profiles and kinematics of "classical" bulges and ellipticals (Kormendy et al. 2008; Hopkins et al. 2008a.i.b. f). The observed kinematics and structural properties of quasar hosts are also consistent with this scenario (Dasyra et al. 2007), and their location on the fundamental plane and associated scaling relations obeyed by classical bulges requires such dissipative star formation in their history (Schweizer 1982; Lake \& Dressler 1986; Hernquist et al. 1993; Dovon et al. 1994; Shier \& Fischer 1998; James et al. 1999; Genzel et al. 2001; Tacconi et al. 2002; Rothberg \& Joseph 2004, 2006; Dasyra et al. 2006, 2007).

\section{DISCUSSION}

In self-regulated scenarios for the growth of black holes, growing the bulge (deepening the central potential around the $\mathrm{BH}$ ) is as much a requirement of sustained bright, high Eddington ratio quasar activity as is actually getting a fuel supply of cold, low angular momentum gas into the vicinity of the $\mathrm{BH}$. Moreover, fueling a Seyfert requires something like the mass of a few giant molecular clouds near the $\mathrm{BH}$, whereas fueling a bright quasar requires channeling a galaxy's worth of cold gas into the central $\sim 10-100 \mathrm{pc}$. This generically leads to the expectation that non-merger related models for quasar fueling become inefficient around a characteristic $\mathrm{BH}$ mass of $\sim 1-3 \times 10^{7} M_{\odot}$ (host bulge mass $\sim 10^{10} M_{\odot}$ ), corresponding to the characteristic bolometric luminosity of these BHs near Eddington, $\sim 10^{12} L_{\odot}-$ remarkably near the traditional Seyfert-quasar divide $\left(M_{B} \approx-23\right.$; Schmidt \& Green 1983). Essentially, this is a restatement of what has become the conventional wisdom and is increasingly established by observation in the field of elliptical galaxy/bulge formation: massive (predominantly "classical") bulges are formed in major mergers, whereas low-mass bulges (predominantly "pseudobulges" in low-mass disks with types $\mathrm{Sb}$ and later) have large populations formed by non-merger mechanisms (i.e. bars and instabilities, or bar-like activity triggered in sufficiently minor 
mergers).

We outline five simple tests to distinguish these modes of fueling and determine whether (and if so where) such a transition occurs. In each case, there is at least tentative evidence for the scenario we outline, although more detailed observations as a function of AGN luminosity and redshift are needed to develop more robust constraints. True quasars tend to have spheroidal hosts, and their relic BHs live in classical bulges; they exhibit characteristically strong small-scale clustering; their redshift evolution from $z \sim 0-2$ is similar to that of the gas-rich merger population; and their colors indicate their nature as post-starburst systems. Seyferts, on the other hand, tend to live in a broader mix of hosts or in disk-dominated galaxies, and their relic BHs exist in pseudo-bulges; they exhibit weaker small-scale clustering, consistent with random galaxies of similar mass; their redshift evolution is weak their abundance remains constant or even decreases with redshift, similar to what is observed in the fraction of barred disks and predicted for stochastic (quiescent) fueling mechanisms; their star formation histories are suggestive of blue, star-forming galaxies.

It is important to emphasize that none of these constraints uniquely or individually implies e.g. a merger origin for bright quasars. For example, the morphologies could reflect their simply being re-ignited by e.g. minor mergers, gas cooling, or stellar mass loss in evolved ellipticals; but this is difficult to reconcile with their clustering (why they do not trace random, more evolved ellipticals in such a case), colors (why they appear post-starburst), or redshift evolution (why they would not grow in abundance at late times, as the cooling rates, stellar mass loss rates, and number density of such ellipticals are all higher). Their classical bulge relics could have been transformed from "initial" pseudo-bulge hosts at the time of the quasars by dissipationless (dry) mergers ${ }^{9}$, but again this fails to explain their clustering and colors, and makes the prediction that there should be very little true classical bulge population at intermediate redshifts. There are many ways to populate galaxies as subhalos that reproduce the clustering signature on small scales (see the discussion in Padmanabhan et al. 2008), but those that correspond to a reasonable fueling hypothesis: e.g. that AGN are random spirals in a certain mass range in groups, or rapidly star-forming objects/LIRGs, fail to reproduce the other observations considered. Redshift evolution and star formation histories are similarly degenerate under various fueling models, but again it is the particular combination of features that is difficult to reproduce.

Together, then, these argue for a scenario with a reasonably rapid (and not strongly redshift-dependent) transition between major merger and non-major merger fueled populations around the traditional Seyfert-quasar divide. If true, this has a number of corresponding implications for the demographics of BHs and AGN: BHs less massive than $\sim 1-3 \times 10^{7} M_{\odot}$ (and their corresponding host bulges $\lesssim 10^{10} M_{\odot}$, typically $\mathrm{Sb}$ and later-type galaxies) would be built up primarily via non-merger mechanisms, whereas more massive black holes and bulges - the "classical" systems generally associated with early-type galaxies (Sa and earlier) - would be built up pri-

\footnotetext{
${ }^{9}$ In practice, this is probably not possible without new dissipation to effectively re-build the bulge, because conservation of phase space densities in dissipationless mergers means that pseudobulges - which characteristically have low central densities corresponding to fitted Sersic indices to their mass profiles with $n_{s} \lesssim 2-$ cannot be dissipationlessly re-merged and produce something with the high central densities characteristic of classical bulges (reflected in Sersic indices $n_{s} \gtrsim 4$ ).
}

marily via mergers. In an integral sense, then, these mergerbuilt systems dominate the total mass density of black holes (with most of the mass density in $\sim 10^{8} M_{\odot}$ black holes near the break in the black hole mass function), constituting $\sim 80 \%$ of the mass in BHs (see e.g. Marconi et al. 2004). Correspondingly (by the Soltan 1982 argument), these systems should dominate the total luminosity density of quasars and AGN (integrated over all redshifts). Given the observed evolution of the quasar luminosity function, a redshiftindependent division at the quasar-Seyfert divide implies that the luminosity density is dominated by mergers at high redshifts, with a transition to dominance of non-merger induced fueling below redshifts $z \sim 1$. Despite their relative lack of importance in the integrated AGN luminosity density, because of the sensitivity to lower-redshift AGN, this suggests that a large (perhaps dominant) fraction of the X-ray background is contributed by these non-merger related systems. There are clearly a large number of important implications for such a distinction.

A major caveat to this comparison is that, depending on the selection depth, wavelength, and methodology, the Seyfert population recovered could have very different properties, biasing it to be more similar to or different from the quasar population. This is because, unlike at bright quasar luminosities which effectively require massive BHs near Eddington, there are two ways to achieve, in practice, Seyfert luminosities: a low-mass $\mathrm{BH}$ at high Eddington ratio and a highmass $\mathrm{BH}$ at low Eddington ratio. High mass systems at low Eddington ratio could be the long-lived remnants of mergers, slowly decaying in AGN luminosity after some initial, bright quasar phase or re-activated by e.g. accretion of new gas in cooling flows or minor mergers, exhibiting characteristically early-type, classical-bulge dominated hosts, with poststarburst populations that might be young for ellipticals but are older than those seen in spiral galaxies. Small-scale clustering signatures in such populations may remain if they were merger-triggered, but it depends significantly on their longterm evolution. The number density evolution of such systems, being cosmologically long-lived and not directly tied to an instantaneous merger rate (even if they are mergertriggered) will reflect, as we predict above, a relatively constant order unity duty cycle. Being at low Eddington ratio, and therefore dim relative to their hosts in optical bands (and possibly radiatively inefficient), such systems may be more commonly seen in X-ray or narrow-line surveys.

On the other hand, surveys of broad-line or optical/IR dominant Seyferts may be more likely to pick out systems at high Eddington ratio, i.e. low-mass, gas-rich systems that might be triggered by minor mergers, bar or disk instabilities, or stochastic encounters with molecular clouds. These systems will be disky, gas-rich, and pseudobulge-dominated, with no small-scale clustering preference, a constant order unity duty cycle, and more ongoing star formation. These distinctions are discussed in detail in Hopkins et al. (2008i); here, we have attempted to develop a robust set of predictions that, together, can distinguish AGN populations connected to mergers from either of these "quiescent" modes of fueling Seyferts (or from the most likely Seyfert population, some mix of the two). But in several of the tests proposed here, these different fueling modes at low luminosities will manifest differently, and thus they should be considered means to constrain and discriminate among different low-luminosity populations, as well. 
We thank Mike Brotherton, Josh Younger, and Pat Hall for helpful conversations and discussion in the development of this paper. This work was supported in part by NSF grants
ACI 96-19019, AST 00-71019, AST 02-06299, and AST 0307690, and NASA ATP grants NAG5-12140, NAG5-13292, and NAG5-13381.

\section{REFERENCES}

Adelberger, K. L., \& Steidel, C. C. 2005, ApJ, 627, L1

Alexander, D. M., et al. 2008, AJ, 135, 1968

Allen, D. A., Roche, P. F., \& Norris, R. P. 1985, MNRAS, 213, 67P

Allen, S. W., Dunn, R. J. H., Fabian, A. C., Taylor, G. B., \& Reynolds, C. S. 2006, MNRAS, 372, 21

Aller, M. C., \& Richstone, D. 2002, AJ, 124, 3035

Aller, M. C., \& Richstone, D. O. 2007, ApJ, 665, 120

Armus, L., Heckman, T., \& Miley, G. 1987, AJ, 94, 831

Athanassoula, E. 2005, MNRAS, 358, 1477

Bahcall, J. N., Kirhakos, S., Saxe, D. H., \& Schneider, D. P. 1997, ApJ, 479, 642

Balcells, M., Graham, A. W., \& Peletier, R. F. 2007, ApJ, 665, 1104

Baldry, I. K., Glazebrook, K., Brinkmann, J., Ivezić, Ž., Lupton, R. H., Nichol, R. C., \& Szalay, A. S. 2004, ApJ, 600, 681

Barazza, F. D., Jogee, S., \& Marinova, I. 2008, ApJ, 675, 1194

Barnes, J. E. 2002, MNRAS, 333, 481

Barnes, J. E., \& Hernquist, L. 1996, ApJ, 471, 115

Barthel, P. D. 2006, A\&A, 458, 107

Barton, E. J., Arnold, J. A., Zentner, A. R., Bullock, J. S., \& Wechsler, R. H. 2007, ApJ, 671, 1538

Baum, S. A., \& McCarthy, P. J. 2000, AJ, 119, 2634

Baum, S. A., O'Dea, C. P., Dallacassa, D., de Bruyn, A. G., \& Pedlar, A. 1993, ApJ, 419, 553

Bell, E. F., Phleps, S., Somerville, R. S., Wolf, C., Borch, A., \& Meisenheimer, K. 2006, ApJ, 652, 270

Bennert, N., Canalizo, G., Jungwiert, B., Stockton, A., Schweizer, F., Peng, C. Y., \& Lacy, M. 2008, ApJ, accepted arXiv:0801.0832 [astro-ph], 801

Best, P. N., von der Linden, A., Kauffmann, G., Heckman, T. M., \& Kaiser, C. R. 2007, MNRAS, 379, 894

Biller, B. A., Jones, C., Forman, W. R., Kraft, R., \& Ensslin, T. 2004, ApJ, 613, 238

Borys, C., Smail, I., Chapman, S. C., Blain, A. W., Alexander, D. M., \& Ivison, R. J. 2005, ApJ, 635, 853

Bournaud, F., Jog, C. J., \& Combes, F. 2005, A\&A, 437, 69

Boylan-Kolchin, M., Ma, C.-P., \& Quataert, E. 2008, MNRAS, 383, 93

Bridge, C. R., et al. 2007, ApJ, 659, 931

Brotherton, M. S., et al. 1999, ApJ, 520, L87

Bundy, K., Ellis, R. S., \& Conselice, C. J. 2005, ApJ, 625, 621

Bundy, K., Fukugita, M., Ellis, R. S., Kodama, T., \& Conselice, C. J. 2004, ApJ, 601, L123

Burkert, A., \& Silk, J. 2001, ApJ, 554, L151

Canalizo, G., \& Stockton, A. 2001, ApJ, 555, 719

Cassata, P., et al. 2005, MNRAS, 357, 903

Cecil, G., Bland-Hawthorn, J., Veilleux, S., \& Filippenko, A. V. 2001, ApJ, 555,338

Colbert, E. J. M., Baum, S. A., Gallimore, J. F., O’Dea, C. P., \& Christensen, J. A. 1996a, ApJ, 467, 551

Colbert, E. J. M., Baum, S. A., Gallimore, J. F., O’Dea, C. P., Lehnert, M. D., Tsvetanov, Z. I., Mulchaey, J. S., \& Caganoff, S. 1996b, ApJS, 105, 75

Colbert, E. J. M., Baum, S. A., O’Dea, C. P., \& Veilleux, S. 1998, ApJ, 496, 786

Combes, F., Debbasch, F., Friedli, D., \& Pfenniger, D. 1990, A\&A, 233, 82

Conselice, C. J. 2006, ApJ, 638, 686

Conselice, C. J., Bershady, M. A., Dickinson, M., \& Papovich, C. 2003, AJ, 126,1183

Cox, T. J., Dutta, S. N., Di Matteo, T., Hernquist, L., Hopkins, P. F., Robertson, B., \& Springel, V. 2006a, ApJ, 650, 791

Cox, T. J., Jonsson, P., Primack, J. R., \& Somerville, R. S. 2006b, MNRAS, 373,1013

Cox, T. J., Jonsson, P., Somerville, R. S., Primack, J. R., \& Dekel, A. 2008, MNRAS, 384, 386

Crenshaw, D. M., et al. 2000, AJ, 120, 1731

Croom, S. M., Boyle, B. J., Shanks, T., Smith, R. J., Miller, L., Outram, P. J., Loaring, N. S., Hoyle, F., \& da Ângela, J. 2005, MNRAS, 356, 415

Croton, D. J. 2006, MNRAS, 369, 1808

da Angela, J., et al. 2008, MNRAS, 383, 565

Dasyra, K. M., Yan, L., Helou, G., Surace, J., Sajina, A., \& Colbert, J. 2008, ApJ, 680, 232

Dasyra, K. M., et al. 2006, ApJ, 638, 745

-. 2007, ApJ, 657, 102 de Kool, M., et al. 2001, ApJ, 548, 609

De Propris, R., Liske, J., Driver, S. P., Allen, P. D., \& Cross, N. J. G. 2005, AJ, 130, 1516

Debattista, V. P., Mayer, L., Carollo, C. M., Moore, B., Wadsley, J., \& Quinn, T. 2006, ApJ, 645, 209

Di Matteo, T., Springel, V., \& Hernquist, L. 2005, Nature, 433, 604

Doyon, R., Wells, M., Wright, G. S., Joseph, R. D., Nadeau, D., \& James,

P. A. 1994, ApJ, 437, L23

Driver, S. P., Allen, P. D., Liske, J., \& Graham, A. W. 2007, ApJ, 657, L85

Driver, S. P., et al. 2006, MNRAS, 368, 414

Drory, N., \& Fisher, D. B. 2007, ApJ, 664, 640

Dunlop, J. S., McLure, R. J., Kukula, M. J., Baum, S. A., O’Dea, C. P., \& Hughes, D. H. 2003, MNRAS, 340, 1095

Elvis, M. 2000, ApJ, 545, 63

Elvis, M., et al. 1994, ApJS, 95, 1

Fakhouri, O., \& Ma, C.-P. 2008, MNRAS, 386, 577

Farrah, D., Afonso, J., Efstathiou, A., Rowan-Robinson, M., Fox, M., \& Clements, D. 2003, MNRAS, 343, 585

Farrah, D., Surace, J. A., Veilleux, S., Sanders, D. B., \& Vacca, W. D. 2005, ApJ, 626, 70

Ferrarese, L., \& Merritt, D. 2000, ApJ, 539, L9

Fine, S., et al. 2006, MNRAS, 373, 613

Fisher, D. B. 2006, ApJ, 642, L17

Fisher, D. B., \& Drory, N. 2008, AJ, in press, arXiv:0805.4206 [astro-ph], 805

Floyd, D. J. E., Kukula, M. J., Dunlop, J. S., McLure, R. J., Miller, L.,

Percival, W. J., Baum, S. A., \& O’Dea, C. P. 2004, MNRAS, 355, 196

Gabel, J. R., Arav, N., \& Kim, T.-S. 2006, ApJ, 646, 742

Gadotti, D. A., \& Kauffmann, G. 2008, MNRAS, in press, arXiv:0811.1219 [astro-ph]

Gebhardt, K., et al. 2000, ApJ, 539, L13

Genzel, R., Hollenbach, D., \& Townes, C. H. 1994, Reports on Progress in Physics, 57, 417

Genzel, R., Tacconi, L. J., Rigopoulou, D., Lutz, D., \& Tecza, M. 2001, ApJ, 563,527

Goto, T. 2005, MNRAS, 357, 937

Graham, A. W., \& Driver, S. P. 2007, ApJ, 655, 77

Graham, A. W., Driver, S. P., Allen, P. D., \& Liske, J. 2007, MNRAS, 378, 198

Graham, A. W., Erwin, P., Caon, N., \& Trujillo, I. 2001, ApJ, 563, L11

Green, P. J. 2006, ApJ, 644, 733

Greene, J. E., Ho, L. C., \& Barth, A. J. 2008, ApJ, 688, 159

Guyon, O., Sanders, D. B., \& Stockton, A. 2006, ApJS, 166, 89

Hasinger, G., Miyaji, T., \& Schmidt, M. 2005, A\&A, 441, 417

Heckman, T. M., Bothun, G. D., Balick, B., \& Smith, E. P. 1984, AJ, 89, 958

Hennawi, J. F., et al. 2006, AJ, 131, 1

Hernquist, L., Spergel, D. N., \& Heyl, J. S. 1993, ApJ, 416, 415

Hibbard, J. E., \& Yun, M. S. 1999, ApJ, 522, L93

Higdon, J. L., Higdon, S. J. U., Willner, S. P., Brown, M. J., Stern, D., Le

Floc'h, E., \& Eisenhardt, P. 2008, ApJ, in press, arXiv:0806.2138 [astro-ph], 806

Hopkins, P. F., Cox, T. J., Dutta, S. N., Hernquist, L., Kormendy, J., \& Lauer, T. R. 2008a, ApJ, accepted, arXiv:0805.3533 [astro-ph], 805

Hopkins, P. F., Cox, T. J., \& Hernquist, L. 2008b, ApJ, accepted, arXiv:0806.3974 [astro-ph], 806

Hopkins, P. F., Cox, T. J., Kereš, D., \& Hernquist, L. 2008c, ApJS, 175, 390

Hopkins, P. F., Cox, T. J., Younger, J. D., \& Hernquist, L. 2008d, ApJ, accepted, arXiv:0806.1739 [astro-ph], 806

Hopkins, P. F., \& Hernquist, L. 2006, ApJS, 166, 1

Hopkins, P. F., Hernquist, L., Cox, T. J., Di Matteo, T., Martini, P., Robertson, B., \& Springel, V. 2005a, ApJ, 630, 705

Hopkins, P. F., Hernquist, L., Cox, T. J., Di Matteo, T., Robertson, B., \& Springel, V. 2005b, ApJ, 630, 716

-. 2005c, ApJ, 632, 81

-. 2006, ApJS, 163, 1

Hopkins, P. F., Hernquist, L., Cox, T. J., Dutta, S. N., \& Rothberg, B. 2008e, ApJ, 679, 156

Hopkins, P. F., Hernquist, L., Cox, T. J., Keres, D., \& Wuyts, S. 2008f, ApJ, accepted, arXiv:0807.2868 [astro-ph], 807

Hopkins, P. F., Hernquist, L., Cox, T. J., \& Kereš, D. 2008g, ApJS, 175, 356 
Hopkins, P. F., Hernquist, L., Cox, T. J., Robertson, B., \& Krause, E. 2007a, ApJ, 669, 45

-. 2007b, ApJ, 669, 67

Hopkins, P. F., Hernquist, L., Cox, T. J., Younger, J. D., \& Besla, G. 2008h, ApJ, accepted, arXiv:0806.2861 [astro-ph], 806

Hopkins, P. F., Hernquist, L., Martini, P., Cox, T. J., Robertson, B., Di Matteo, T., \& Springel, V. 2005d, ApJ, 625, L71

Hopkins, P. F., Lauer, T. R., Cox, T. J., Hernquist, L., \& Kormendy, J. 2008i, ApJ, in press, arXiv:0806.2325 [astro-ph], 806

Hopkins, P. F., Lidz, A., Hernquist, L., Coil, A. L., Myers, A. D., Cox, T. J., \& Spergel, D. N. 2007c, ApJ, 662, 110

Hopkins, P. F., Richards, G. T., \& Hernquist, L. 2007d, ApJ, 654, 731

Hopkins, P. F., et al. 2008j, ApJ, in prep

Hu, J. 2008, MNRAS, 386, 2242

Hutchings, J. B., Cherniawsky, A., Cutri, R. M., \& Nelson, B. O. 2006, AJ, 131,680

Hutchings, J. B., Maddox, N., Cutri, R. M., \& Nelson, B. O. 2003, AJ, 126, 63

Hutchings, J. B., \& Neff, S. G. 1992, AJ, 104, 1

Jahnke, K., Kuhlbrodt, B., \& Wisotzki, L. 2004a, MNRAS, 352, 399

Jahnke, K., et al. 2004b, ApJ, 614, 568

James, P., Bate, C., Wells, M., Wright, G., \& Doyon, R. 1999, MNRAS, 309,585

Jogee, S. 2004, in AGN Physics on All Scales, Lect.Notes Phys. 693 (2006), 143

Jogee, S., et al. 2004, ApJ, 615, L105

Joseph, R. D., \& Wright, G. S. 1985, MNRAS, 214, 87

Kartaltepe, J. S., et al. 2007, ApJS, 172, 320

Kaspi, S., et al. 2002, ApJ, 574, 643

Kauffmann, G., et al. 2003, MNRAS, 346, 1055

Kawakatu, N., Anabuki, N., Nagao, T., Umemura, M., \& Nakagawa, T. 2006, ApJ, 637, 104

Kewley, L. J., Groves, B., Kauffmann, G., \& Heckman, T. 2006, MNRAS, 372,961

Kim, M., Ho, L. C., Peng, C. Y., Barth, A. J., Im, M., Martini, P., \& Nelson, C. H. 2008, ApJ, in press, arXiv:0807.1337 [astro-ph], 807

Kochanek, C. S., et al. 2001, ApJ, 560, 566

Kormendy, J., Fisher, D. B., Cornell, M. E., \& Bender, R. 2008, ApJ, in press, arXiv:0810.1681 [astro-ph]

Kormendy, J., \& Kennicutt, Jr., R. C. 2004, ARA\&A, 42, 603

Kormendy, J., \& Richstone, D. 1995, ARA\&A, 33, 581

Kormendy, J., \& Sanders, D. B. 1992, ApJ, 390, L53

Krause, E., et al. 2008, ApJ, in preparation

Krongold, Y., Nicastro, F., Elvis, M., Brickhouse, N., Binette, L., Mathur, S., \& Jiménez-Bailón, E. 2007, ApJ, 659, 1022

Lake, G., \& Dressler, A. 1986, ApJ, 310, 605

Laor, A., Fiore, F., Elvis, M., Wilkes, B. J., \& McDowell, J. C. 1997, ApJ, 477, 93

Le Floc'h, E., et al. 2005, ApJ, 632, 169

Levenson, N. A., Weaver, K. A., \& Heckman, T. M. 2001, ApJ, 550, 230

Lin, L., et al. 2004, ApJ, 617, L9

—. 2008, ApJ, in press, arXiv:0802.3004 [astro-ph], 802

Lotz, J. M., Jonsson, P., Cox, T. J., \& Primack, J. R. 2008a, MNRAS, in press, arXiv:0805.1246 [astro-ph], 805

Lotz, J. M., Madau, P., Giavalisco, M., Primack, J., \& Ferguson, H. C. 2006, ApJ, 636, 592

Lotz, J. M., et al. 2008b, ApJ, 672, 177

Lutz, D., et al. 2008, ApJ, in press, arXiv:0805.2669 [astro-ph], 805

Magorrian, J., et al. 1998, AJ, 115, 2285

Malkan, M. A., Gorjian, V., \& Tam, R. 1998, ApJS, 117, 25

Maller, A. H., Katz, N., Kereš, D., Davé, R., \& Weinberg, D. H. 2006, ApJ, 647, 763

Marconi, A., \& Hunt, L. K. 2003, ApJ, 589, L21

Marconi, A., Risaliti, G., Gilli, R., Hunt, L. K., Maiolino, R., \& Salvati, M. 2004, MNRAS, 351, 169

Marinova, I., \& Jogee, S. 2007, ApJ, 659, 1176

Martini, P. 2004, in Coevolution of Black Holes and Galaxies, ed. L. C. Ho, 169

McKernan, B., Yaqoob, T., \& Reynolds, C. S. 2007, MNRAS, 379, 1359

Merloni, A., Rudnick, G., \& Di Matteo, T. 2004, MNRAS, 354, L37

Mihos, J. C., \& Hernquist, L. 1994a, ApJ, 437, L47

-. 1994b, ApJ, 431, L9

-. 1996, ApJ, 464, 641

Murray, N., Quataert, E., \& Thompson, T. A. 2005, ApJ, 618, 569

Myers, A. D., Brunner, R. J., Richards, G. T., Nichol, R. C., Schneider, D. P., \& Bahcall, N. A. 2007, ApJ, 658, 99

Naab, T., \& Burkert, A. 2003, ApJ, 597, 893
Naab, T., Jesseit, R., \& Burkert, A. 2006, MNRAS, 372, 839

Naab, T., Johansson, P. H., Ostriker, J. P., \& Efstathiou, G. 2007, ApJ, 658 , 710

Nandra, K., et al. 2007, ApJ, 660, L11

O’Neill, J. K., \& Dubinski, J. 2003, MNRAS, 346, 251

Padmanabhan, N., et al. 2008, ApJ, in preparation

Patton, D. R., et al. 2002, ApJ, 565, 208

Peng, C. Y., Impey, C. D., Rix, H.-W., Kochanek, C. S., Keeton, C. R.,

Falco, E. E., Lehár, J., \& McLeod, B. A. 2006, ApJ, 649, 616

Pierce, C. M., et al. 2007, ApJ, 660, L19

Porciani, C., \& Norberg, P. 2006, MNRAS, 371, 1824

Pounds, K. A., King, A. R., Page, K. L., \& O'Brien, P. T. 2003a, MNRAS, 346,1025

Pounds, K. A., Reeves, J. N., King, A. R., Page, K. L., O’Brien, P. T., \& Turner, M. J. L. 2003b, MNRAS, 345, 705

Reddy, N. A., Steidel, C. C., Fadda, D., Yan, L., Pettini, M., Shapley, A. E., Erb, D. K., \& Adelberger, K. L. 2006, ApJ, 644, 792

Rice, M. S., Martini, P., Greene, J. E., Pogge, R. W., Shields, J. C.,

Mulchaey, J. S., \& Regan, M. W. 2006, ApJ, 636, 654

Richards, G. T., et al. 2006, ApJS, 166, 470

Rigby, J. R., Rieke, G. H., Donley, J. L., Alonso-Herrero, A., \& Pérez-González, P. G. 2006, ApJ, 645, 115

Robertson, B., Bullock, J. S., Cox, T. J., Di Matteo, T., Hernquist, L., Springel, V., \& Yoshida, N. 2006a, ApJ, 645, 986

Robertson, B., Cox, T. J., Hernquist, L., Franx, M., Hopkins, P. F., Martini, P., \& Springel, V. 2006b, ApJ, 641, 21

Rothberg, B., \& Joseph, R. D. 2004, AJ, 128, 2098

-. 2006, AJ, 131, 185

Ruiz, J. R., et al. 2001, AJ, 122, 2961

Rupke, D. S., Veilleux, S., \& Sanders, D. B. 2005, ApJ, 632, 751

Sajina, A., et al. 2008, ApJ, in press, arXiv:0805.0263 [astro-ph], 805

Salpeter, E. E. 1955, ApJ, 121, 161

-. 1964, ApJ, 140, 796

Salucci, P., Szuszkiewicz, E., Monaco, P., \& Danese, L. 1999, MNRAS, 307, 637

Sánchez, S. F., et al. 2004, ApJ, 614, 586

Sanders, D. B., \& Mirabel, I. F. 1996, ARA\&A, 34, 749

Sanders, D. B., Soifer, B. T., Elias, J. H., Madore, B. F., Matthews, K., Neugebauer, G., \& Scoville, N. Z. 1988a, ApJ, 325, 74

Sanders, D. B., Soifer, B. T., Elias, J. H., Neugebauer, G., \& Matthews, K 1988b, ApJ, 328, L35

Sargent, A. I., Sanders, D. B., Scoville, N. Z., \& Soifer, B. T. 1987, ApJ, 312, L35

Scannapieco, E., \& Oh, S. P. 2004, ApJ, 608, 62

Schmidt, M., \& Green, R. F. 1983, ApJ, 269, 352

Schweizer, F. 1982, ApJ, 252, 455

Scoville, N. Z., Sanders, D. B., Sargent, A. I., Soifer, B. T., Scott, S. L., \& Lo, K. Y. 1986, ApJ, 311, L47

Serber, W., Bahcall, N., Ménard, B., \& Richards, G. 2006, ApJ, 643, 68

Shankar, F., Bernardi, M., \& Haiman, Z. 2008, ApJ, in press, arXiv:0806.3459 [astro-ph], 806

Shankar, F., Weinberg, D. H., \& Miralda-Escude', J. 2007, ApJ, in press, arXiv:0710.4488 [astro-ph], 710

Shen, Y., et al. 2007, AJ, 133, 2222

Sheth, K., Regan, M. W., Scoville, N. Z., \& Strubbe, L. E. 2003, ApJ, 592, L13

Shi, Y., et al. 2007, ApJ, 669, 841

Shields, G. A., Gebhardt, K., Salviander, S., Wills, B. J., Xie, B.,

Brotherton, M. S., Yuan, J., \& Dietrich, M. 2003, ApJ, 583, 124

Shier, L. M., \& Fischer, J. 1998, ApJ, 497, 163

Silk, J., \& Rees, M. J. 1998, A\&A, 331, L1

Silverman, J. D., et al. 2008, ApJ, 675, 1025

Soifer, B. T., Sanders, D. B., Madore, B. F., Neugebauer, G., Danielson,

G. E., Elias, J. H., Lonsdale, C. J., \& Rice, W. L. 1987, ApJ, 320, 238 Soltan, A. 1982, MNRAS, 200, 115

Springel, V., Di Matteo, T., \& Hernquist, L. 2005a, ApJ, 620, L79

-. 2005b, MNRAS, 361, 776

Springel, V., \& Hernquist, L. 2005, ApJ, 622, L9

Stark, A. A., \& Carlson, E. R. 1984, ApJ, 279, 122

Steenbrugge, K. C., et al. 2005, A\&A, 434, 569

Stewart, K. R., Bullock, J. S., Wechsler, R. H., Maller, A. H., \& Zentner,

A. R. 2007, ApJ, in press arXiv:0711.5027 [astro-ph], 711

Stockton, A. 1978, Nature, 274, 342

Stockton, A., \& MacKenty, J. W. 1987, ApJ, 316, 584

Stockton, A., \& Ridgway, S. E. 1991, AJ, 102, 488

Strateva, I., et al. 2001, AJ, 122, 1861 
Tacconi, L. J., Genzel, R., Lutz, D., Rigopoulou, D., Baker, A. J., Iserlohe, C., \& Tecza, M. 2002, ApJ, 580, 73

Thacker, R. J., Scannapieco, E., \& Couchman, H. M. P. 2006, ApJ, 653, 86

Thompson, T. A., Quataert, E., \& Murray, N. 2005, ApJ, 630, 167

Tremaine, S., et al. 2002, ApJ, 574, 740

Urrutia, T., Lacy, M., \& Becker, R. H. 2008, ApJ, 674, 80

Vanden Berk, D. E., et al. 2006, AJ, 131, 84

Veilleux, S., Cecil, G., \& Bland-Hawthorn, J. 2005, ARA\&A, 43, 769

Walter, F., Carilli, C., Bertoldi, F., Menten, K., Cox, P., Lo, K. Y., Fan, X., \& Strauss, M. A. 2004, ApJ, 615, L17

Walter, F., Weiss, A., \& Scoville, N. 2002, ApJ, 580, L21

Wang, R., et al. 2008, ApJ, in press, arXiv:0806.3022 [astro-ph], 806

Weymann, R. J., Carswell, R. F., \& Smith, M. G. 1981, ARA\&A, 19, 41

Whittle, M., \& Wilson, A. S. 2004, AJ, 127, 606

Wolf, C., et al. 2005, ApJ, 630, 771
Woo, J.-H., Treu, T., Malkan, M. A., \& Blandford, R. D. 2006, ApJ, 645, 900 Woods, D. F., \& Geller, M. J. 2007, AJ, 134, 527

Woods, D. F., Geller, M. J., \& Barton, E. J. 2006, AJ, 132, 197

Wyithe, J. S. B., \& Loeb, A. 2002, ApJ, 581, 886

Xu, C. K., Sun, Y. C., \& He, X. T. 2004, ApJ, 603, L73

Yan, L., Sajina, A., Fadda, D., Choi, P., Armus, L., Helou, G., Teplitz, H., Frayer, D., \& Surace, J. 2007, ApJ, 658, 778

Yip, C. W., et al. 2004, AJ, 128, 2603

Younger, J. D., Hopkins, P. F., Cox, T. J., \& Hernquist, L. 2008, ApJ, in press, arXiv:0804.2672 [astro-ph], 804

Yu, Q., \& Tremaine, S. 2002, MNRAS, 335, 965

Zakamska, N. L., et al. 2006, AJ, 132, 1496

-. 2008, ApJ, in preparation 